\title{
Obstáculos enfrentados pela Enfermagem na prevenção de infecções sexualmente transmissíveis na terceira idade
}

\author{
Obstacles faced by Nursing in the prevention of sexually transmitted infections in the \\ elderly
}

\begin{abstract}
Obstáculos enfrentados por la Enfermería en la prevención de infecciones de transmisión sexual en la tercera edad
\end{abstract}

Marlúcia de Sousa Rodrigues ${ }^{1}$, Maria Nauside Pessoa da Silva ${ }^{1,2}$, Francisco Lucas de Lima Fontes $^{1 *}$, Vanessa Maria Oliveira Viana ${ }^{2}$, Maria da Cruz Silva Pessoa Santos ${ }^{1}$, Francisca Jéssica Abreu da Silva ${ }^{1}$, Janaina Ribeiro Corado ${ }^{1}$, Ariane Freire Oliveira ${ }^{3}$, Onédia Naís de Carvalho ${ }^{3}$, Luana Letícia Silva Carvalho ${ }^{3}$, Tágila Andreia Viana dos Santos ${ }^{4}$, Maurício José Almeida Morais ${ }^{1}$, Jakson de Oliveira Gaia ${ }^{1}$, Luan da Silva Morais ${ }^{3}$, Sandra Maria Gomes de Sousa ${ }^{5}$.

\section{RESUMO}

Objetivo: Identificar os obstáculos enfrentados pela Enfermagem na prevenção de infecções sexualmente transmissíveis na terceira idade. Métodos: Revisão integrativa com abordagem qualitativa. Foi desenvolvida por meio da biblioteca virtual Scientific Electronic Library Online, da base de dados Literatura LatinoAmericana e do Caribe em Ciências da Saúde e do buscador virtual Google Acadêmico, selecionando-se 12 artigos para construção da discussão. Resultados: A perpetuação de padrões arcaicos sobre a sexualidade, falta de conhecimento dos idosos, falhas nas ações educativas da equipe de Enfermagem e a resistência do sujeito idoso quanto ao uso do preservativo foram alguns dos obstáculos encontrados. Considerações finais: A Enfermagem, por ser uma profissão que lida com o paciente nos três níveis de atenção à saúde, deve adotar estratégias de ações educativas singulares apropriadas ao grau de conhecimento do idoso que está sendo atendido, de modo a construir uma discussão baseada nas informações prévias que o sujeito possui acerca das infecções sexualmente transmissíveis. Acolhimento, criação de vínculo, atendimento holístico e diálogo emergem como estratégias importantes na edificação de uma relação profissional-paciente de confiança, possibilitando adesão às práticas orientadas pelo profissional de Enfermagem.

Palavras-chave: Enfermagem, Idosos, Infecções sexualmente transmissíveis.

\footnotetext{
ABSTRACT

Objective: To identify the obstacles faced by Nursing in the prevention of sexually transmitted infections in the elderly. Methods: Integrative review with a qualitative approach. It was developed through the Scientific Electronic Library Online virtual library, the Latin American and Caribbean Literature in Health Sciences database and the Google Scholar virtual search engine, selecting 12 articles to build the discussion. Results: The perpetuation of archaic patterns about sexuality, lack of knowledge of the elderly, flaws in the educational actions of the nursing team and the resistance of the elderly subject regarding the use of condoms were some

${ }^{1}$ Faculdade UNINASSAU - Campus Redenção. Teresina, Piauí, Brasil. *E-mail:lucasfontesenf@hotmail.com

2 Universidade Federal do Piauí. Teresina, Piauí, Brasil

${ }^{3}$ Centro Universitário UNINOVAFAPI. Teresina, Piauí, Brasil.

4 Unidades Integradas de Pós-graduação. Teresina, Piauí, Brasil.

5 Universidade Estadual do Piauí. Teresina, Piauí, Brasil.
} 
of the obstacles encountered. Final considerations: The Nursing, as a profession that deals with the patient in the three levels of health care, should adopt singular educational strategies appropriate to the degree of knowledge of the elderly being served, in order to construct a discussion based on the information the subject has about sexually transmitted infections. Welcoming, bonding, holistic care and dialogue emerge as important strategies in building a trustful professional-patient relationship, making possible adherence to practices guided by the Nursing professional.

Keywords: Nursing, Elderly, Sexually transmitted infections.

\section{RESUMEN}

Objetivo: Identificar los obstáculos enfrentados por la Enfermería en la prevención de infecciones sexualmente transmisibles en la tercera edad. Métodos: Revisión integrativa con enfoque cualitativo. Se desarrolló a través de la biblioteca virtual Scientific Electronic Library Online, de la base de datos Literatura Latinoamericana y del Caribe en Ciencias de la Salud y del buscador virtual Google Académico, seleccionando 12 artículos para la construcción de la discusión. Resultados: La perpetuación de patrones arcaicos sobre la sexualidad, falta de conocimiento de los ancianos, fallas en las acciones educativas del equipo de Enfermería y la resistencia del sujeto mayor en cuanto al uso del preservativo fueron algunos de los obstáculos encontrados. Consideraciones finales: La Enfermería, por ser una profesión que lidia con el paciente en los tres niveles de atención a la salud, debe adoptar estrategias de acciones educativas singulares apropiadas al grado de conocimiento del anciano que está siendo atendido, para construir una discusión basada en la información previas que el sujeto posee acerca de las infecciones de transmisión sexual. Acogida, creación de vínculo, atención holística y diálogo emergen como estrategias importantes en la edificación de una relación profesional-paciente de confianza, posibilitando adhesión a las prácticas orientadas por el profesional de Enfermería.

Palabras-clave: Enfermería, Ancianos, Infecciones de transmisión sexual.

\section{INTRODUÇÃO}

A sexualidade é considerada uma forma íntima de expressão do ser humano, identificada de maneira global na espécie. Relaciona-se a variadas vertentes como na educação, ao estudar o corpo e o manejo da sociedade por meio de condutas sexuais, e também na saúde pública, ao envolver-se com a natalidade e a vitalidade das descendências e da espécie (FIGUEIROA MN, et al., 2017).

Envolve diversos aspectos na vida do sujeito: identidade, gênero, orientação sexual, prazer, reprodução, erotismo e intimidade. Por vezes, tais dimensões são influenciadas por crenças, valores e comportamentos que associam-se às ordens biológica, mental, religiosa e espiritual (VIEIRA KFL, et al., 2016).

Os obstáculos na aceitação da sexualidade na dinâmica do envelhecimento perpassam pela falta de informação, provavelmente pela ideia arcaica de que a sexualidade limita-se apenas ao uso do aparelho genital e à reprodução. Contudo, percebe-se nos últimos anos, que a terceira idade tem estreitado vínculos com a vida sexual ativa, o que é essencial à boa qualidade de vida, mas também coloca os sujeitos dessa faixa etária em maior predisposição à aquisição de infecções sexualmente transmissíveis (ISTs) (ASSIS CL e FILHO JS, 2015).

Um estudo de revisão sobre a epidemiologia das ISTs na população idosa evidenciou taxas elevadas dessas infecções em países da América do Norte, Austrália, China, Coreia e África Subsaariana (MINICHIELLO V, et al., 2012).

No Brasil há uma carência de dados nacionais acerca da prevalência das ISTs em geral e em sujeitos da terceira idade, muito provavelmente por muitas delas não serem de notificação compulsória. Para o Vírus da Imunodeficiência Humana (HIV), contudo, os boletins publicados mostram aumento importante de casos entre 
homens e mulheres na faixa acima de 60 anos na última década, apontando para a vulnerabilidade desse segmento da sociedade às infecções (ANDRADE J, et al., 2017).

O Brasil acompanha uma tendência mundial de envelhecimento populacional, com aumento do quantitativo de idosos, incluindo àqueles com ISTs. O aumento dessas infecções na terceira idade pode relacionar-se ao desenvolvimento de recursos e medicamentos que favorecem o desempenho sexual, como o uso de prótese para tratamento de disfunção erétil, fármacos estimulantes para homens e regulação hormonal para mulheres (SOUZA MP, et al., 2015).

Devido ao aumento das ISTs na terceira idade, o Estado deve prover políticas públicas de enfretamento a essas infecções por meio de medidas preventivas. É essencial que o idoso tenha acesso a informações sobre sexualidade, infecções transmitidas por meio do ato sexual, bem como das repercussões que essas infecções podem trazer a sua vida e a sua saúde (CASTRO SFF, et al., 2014).

Nesse sentido, os profissionais de Enfermagem devem possuir tato para envolver o sujeito idoso com a temática. É necessária devida capacitação desses profissionais para que a abordagem seja efetiva, englobando acolhimento, escuta qualificada e repasse de informações úteis para a prevenção de infecções causadas pelo ato sexual desprotegido (CASSÉTTE JB, et al., 2016).

A justificativa para realização deste estudo decorre de inquietações sobre como ocorre a assistência de Enfermagem ao indivíduo idoso predisposto à aquisição de IST. Poderá contribuir na identificação das estratégias utilizadas pelo profissional de Enfermagem no atendimento ao sujeito com vida sexual ativa na terceira idade, de modo a sensibilizar os indivíduos na adesão às práticas preventivas de ISTs e na valorização da saúde do corpo como condição necessária para que esse grupo de pessoas possa usufruir da melhor forma possível.

Diante da breve explanação, o objetivo do presente estudo foi identificar os obstáculos enfrentados pela Enfermagem na prevenção de infecções sexualmente transmissíveis na terceira idade.

\section{MÉTODOS}

Trata-se de uma revisão integrativa com abordagem qualitativa que levantou a seguinte questão norteadora: "Quais os obstáculos enfrentados pela equipe de Enfermagem na prevenção de infecções sexualmente transmissíveis na terceira idade?".

Foi desenvolvida por meio da biblioteca virtual Scientific Electronic Library Online (SciELO), da base de dados Literatura Latino-Americana e do Caribe em Ciências da Saúde (LILACS) e do buscador virtual Google Acadêmico, utilizando-se como critérios de inclusão: artigos originais disponíveis na íntegra, publicados entre 2014 e 2019, em língua portuguesa e que versassem sobre o objeto de estudo desta revisão. Foram excluídas monografias, dissertações e teses.

Utilizaram-se os seguintes Descritores em Ciência da Saúde (DeCS) para a pesquisa na literatura: "Enfermagem", "terceira idade", "prevenção" e "infecções sexualmente transmissíveis". A análise do material coletado ocorreu com base na leitura e interpretação das informações teóricas e dos resultados encontrados em cada publicação selecionada. Após aplicação dos critérios estabelecidos e análise de conteúdo, foram selecionados 12 artigos para construção da discussão.

\section{RESULTADOS E DISCUSSÃO}

Ao analisar os critérios de inclusão e exclusão citados nos procedimentos metodológicos para a seleção dos artigos, foi possível elaborar um quadro para melhor compreensão dos dados. Foram estabelecidas cinco variáveis que são relevantes para os resultados (autor/ano, título, tipo de estudo, amostra e conclusão) como estratégia para a elaboração da discussão baseada nas produções científicas da temática pesquisada, conforme se observa no Quadro 1. 
Quadro 1 - Distribuição das produções científicas segundo autor/ano, título, tipo de estudo, amostra e conclusão. Teresina, Piauí, Brasil, 2019.

\begin{tabular}{|c|c|c|c|c|}
\hline Autor/Ano & Título & Tipo de estudo & Amostra & Conclusão \\
\hline $\begin{array}{l}\text { SILVA AT et } \\
\text { al. (2019) }\end{array}$ & $\begin{array}{l}\text { Prevalência da AIDS em } \\
\text { idosos no centro-oeste } \\
\text { brasileiro }\end{array}$ & $\begin{array}{c}\text { Estudo } \\
\text { quantitativo }\end{array}$ & 1.184 casos & $\begin{array}{l}\text { Há um número considerável de } \\
\text { idosos sexualmente ativos, } \\
\text { mostrando que o estigma de } \\
\text { população assexuada precisa ser } \\
\text { desconsiderado, e é necessário } \\
\text { criar ações em saúde direcionadas } \\
\text { a essa faixa etária. }\end{array}$ \\
\hline $\begin{array}{l}\text { CAETANO } \\
\text { KS et al. } \\
(2018)\end{array}$ & $\begin{array}{l}\text { HIV/AIDS: conhecimento, } \\
\text { atitude e prática da pessoa } \\
\text { idosa }\end{array}$ & $\begin{array}{c}\text { Estudo } \\
\text { quantitativo }\end{array}$ & 72 idosos & $\begin{array}{l}\text { A população idosa se sente carente } \\
\text { de informações e atendimentos que } \\
\text { retratam especificamente temáticas } \\
\text { relacionadas ao HIV/AIDS, a fim de } \\
\text { oportunizar para que estes } \\
\text { indivíduos tenham conhecimento, } \\
\text { atitude e práticas adequadas. }\end{array}$ \\
\hline $\begin{array}{l}\text { MALAQUIAS } \\
\text { BSS et al. } \\
(2017)\end{array}$ & $\begin{array}{l}\text { Pesquisa com idosos sobre } \\
\text { HIV/AIDS e sexualidade: } \\
\text { relato de experiência }\end{array}$ & $\begin{array}{c}\text { Relato de } \\
\text { experiência }\end{array}$ & 457 idosos & $\begin{array}{c}\text { Muitos idosos ficam sem acesso a } \\
\text { informações pela falta de } \\
\text { oportunidades em conversar sobre } \\
\text { o assunto, que ainda é algo } \\
\text { banalizado. }\end{array}$ \\
\hline $\begin{array}{l}\text { SILVA JDB } \\
\text { et al. (2017) }\end{array}$ & $\begin{array}{c}\text { Vulnerabilidade às infecções } \\
\text { sexualmente } \\
\text { transmissíveis/AIDS em } \\
\text { idosos }\end{array}$ & $\begin{array}{c}\text { Estudo } \\
\text { quantitativo }\end{array}$ & 40 idosos & $\begin{array}{l}\text { Observa-se que a sexualidade deve } \\
\text { ser debatida com os idosos e } \\
\text { estimulada dentro de uma prática } \\
\text { saudável e sem estigmas. }\end{array}$ \\
\hline $\begin{array}{l}\text { BRITO NMI } \\
\text { et al. (2016) }\end{array}$ & $\begin{array}{c}\text { Idosos, infecções } \\
\text { sexualmente transmissíveis } \\
\text { e AIDS: conhecimentos e } \\
\text { percepção de risco }\end{array}$ & $\begin{array}{c}\text { Estudo } \\
\text { quantitativo }\end{array}$ & 55 idosos & $\begin{array}{c}\text { Cabe aos organismos } \\
\text { governamentais e não } \\
\text { governamentais investir em práticas } \\
\text { educativas, onde idosos possam } \\
\text { ser inseridos em um ambiente que } \\
\text { aborde a sexualidade, } \\
\text { proporcionando maior segurança e } \\
\text { qualidade de vida aos nossos } \\
\text { cidadãos. }\end{array}$ \\
\hline $\begin{array}{l}\text { OLIVEIRA } \\
\text { JMS e } \\
\text { CÂNDIDO } \\
\text { ASC (2016) }\end{array}$ & $\begin{array}{l}\text { Conhecimento dos idosos } \\
\text { sobre as medidas de } \\
\text { prevenção das DST's }\end{array}$ & Estudo qualitativo & 6 idosos & $\begin{array}{l}\text { Viu-se que os grupos de } \\
\text { convivência são cruciais e, no caso } \\
\text { dos idosos entrevistados, ajudou-os } \\
\text { a serem informados em relação as } \\
\text { DSTs, trazendo muitos outros } \\
\text { benefícios diretos e indiretos; } \\
\text { nestes o profissional de saúde tem } \\
\text { a possibilidade de orientar da } \\
\text { melhor forma possível, garantindo- } \\
\text { Ihes o bem-estar, a saúde e } \\
\text { qualidade de vida. }\end{array}$ \\
\hline $\begin{array}{l}\text { BEZERRA } \\
\text { VP et al. } \\
(2015)\end{array}$ & $\begin{array}{c}\text { Práticas preventivas de } \\
\text { idosos e a vulnerabilidade ao } \\
\text { HIV }\end{array}$ & Estudo qualitativo & 37 idosos & $\begin{array}{c}\text { A complexidade das variadas } \\
\text { dimensões experimentadas pelos } \\
\text { idosos estudados indica a } \\
\text { necessidade de realização de } \\
\text { outros estudos que possibilitem } \\
\text { progressos no entendimento da } \\
\text { subjetividade colocada nas relações } \\
\text { que perpassam o processo de } \\
\text { envelhecimento e a experiencia da } \\
\text { sexualidade nessa população. }\end{array}$ \\
\hline
\end{tabular}




\begin{tabular}{|c|c|c|c|c|}
\hline $\begin{array}{l}\text { CUNHA LM } \\
\text { et al. (2015) }\end{array}$ & $\begin{array}{c}\text { Vovó e vovô também amam: } \\
\text { sexualidade na terceira } \\
\text { idade }\end{array}$ & Estudo qualitativo & $\begin{array}{c}12 \\
\text { profissionais }\end{array}$ & $\begin{array}{l}\text { O tema era de difícil abordagem } \\
\text { durante as consultas, embora } \\
\text { relevante no contexto das unidades } \\
\text { de saúde. Ressalta-se a escassez } \\
\text { de ações voltadas para essa } \\
\text { temática nas unidades de saúde, } \\
\text { sinalizando grande fragilidade no } \\
\text { que diz respeito à atenção integral à } \\
\text { saúde do idoso. }\end{array}$ \\
\hline $\begin{array}{l}\text { MOREIRA } \\
\text { WC et al. } \\
(2015)\end{array}$ & $\begin{array}{c}\text { Sexualidade e prevenção de } \\
\text { IST e HIV/aids entre idosos } \\
\text { usuários da estratégia saúde } \\
\text { da família }\end{array}$ & $\begin{array}{c}\text { Relato de } \\
\text { experiência }\end{array}$ & $\begin{array}{c}\text { Não } \\
\text { especificado }\end{array}$ & $\begin{array}{c}\text { Faz-se necessário a criação de } \\
\text { políticas mais eficazes e, sobretudo } \\
\text { uma conscientização dos } \\
\text { profissionais da saúde sobre a } \\
\text { necessidade de planejar, orientar e } \\
\text { implementar ações nesta temática } \\
\text { no intuito de empoderar esses } \\
\text { idosos. }\end{array}$ \\
\hline $\begin{array}{l}\text { PEIXER TC } \\
\text { et al. (2015) }\end{array}$ & $\begin{array}{c}\text { Sexualidade na terceira } \\
\text { idade: percepção de homens } \\
\text { idosos de uma estratégia de } \\
\text { saúde da família }\end{array}$ & Estudo qualitativo & 5 idosos & $\begin{array}{l}\text { Há limitações relacionadas à } \\
\text { discussão sobre sexualidade na } \\
\text { terceira idade. Além disso, os } \\
\text { profissionais de saúde estão } \\
\text { poucos presentes na orientação e } \\
\text { esclarecimento de fatores } \\
\text { relacionados à sexualidade. }\end{array}$ \\
\hline $\begin{array}{l}\text { BEZERRA } \\
\text { VP et al. } \\
(2014)\end{array}$ & $\begin{array}{c}\text { Vulnerabilidade de idosos ao } \\
\text { contágio pelo HIV no } \\
\text { contexto de práticas } \\
\text { preventivas }\end{array}$ & $\begin{array}{c}\text { Estudo } \\
\text { quantitativo }\end{array}$ & 84 idosos & $\begin{array}{l}\text { Idosos apresentam vulnerabilidade } \\
\text { ao contágio pelo HIV ao mostrar } \\
\text { práticas sexuais que dispensam o } \\
\text { uso de preservativo sendo } \\
\text { recomendadas ações educativas de } \\
\text { prevenção frente ao HIV. }\end{array}$ \\
\hline $\begin{array}{l}\text { CASTRO } \\
\text { SFF et al. } \\
(2014)\end{array}$ & $\begin{array}{c}\text { Prevenção da AIDS em } \\
\text { idosos: visão e prática do } \\
\text { enfermeiro }\end{array}$ & Estudo qualitativo & $\begin{array}{c}10 \\
\text { enfermeiros }\end{array}$ & $\begin{array}{l}\text { Observou-se que há um } \\
\text { preconceito, ancorado em questões } \\
\text { de cunho cultural, por parte do } \\
\text { enfermeiro sobre a sexualidade do } \\
\text { idoso, e isso consequentemente } \\
\text { reflete na falta de ações e } \\
\text { estratégias eficazes de prevenção } \\
\text { da AIDS nessa faixa etária. }\end{array}$ \\
\hline
\end{tabular}

Fonte: Dados da pesquisa (2019).

A sexualidade na terceira idade é algo possível, porém muitas vezes dificultada pela sociedade por meio da imposição de padrões e estereótipos engendrados culturalmente. Esses obstáculos enfrentados pelo idoso relacionam-se a princípios e valores obtidos em uma educação severa, onde pais orientavam seus filhos de maneira repressora, levando-os a compreender a sexualidade como sinônimo de promiscuidade, o que pode reverberar no autoestigma que o próprio sujeito idoso sente ao tocar no assunto (CASTRO SFF, et al., 2014).

Ainda vista como um tabu por diversos profissionais, a sexualidade na terceira idade é frequente alvo de preconceito. O estudo de Malaquias BSS, et al. (2017) constatou falhas nas ações educativas da equipe de Enfermagem voltadas à prevenção de ISTs na terceira idade que, por vezes, são poucas ou inexistentes. Nesse sentido, é de fundamental importância reforçar aos profissionais o envelhecimento como algo natural ao ser humano e a sexualidade como dimensão necessária ao envelhecimento ativo, visando a prestação de uma assistência holística ao sujeito idoso.

As consultas profissionais podem surgir como recurso facilitador ou dificultador nas abordagens preventivas às ISTs. O estudo de Cunha LM, et al. (2015) mostrou discrepâncias no atendimento de médico 
e enfermeiro acerca das necessidades sexuais da população idosa. Enquanto o primeiro ator volta sua assistência à dimensão curativa e terapêutica, o enfermeiro assume a sexualidade com enfoque mais subjetivo, privilegiando a discussão de dúvidas, diálogo e norteio.

Bezerra VP, et al. (2014) apontaram que os profissionais de Enfermagem devem utilizar o diálogo como principal estratégia na busca de um ambiente propicio à discussão das relações sexuais protegidas. Os resultados do estudo mostraram que a realização de ações educativas, de maneira integral pela Enfermagem, bem como a implementação de ações como orientações, criação de vínculos e receptividade no atendimento, ajudam na adesão às práticas preventivas e consequentemente reduzem o número de casos de ISTs e infecção pelo HIV nessa faixa etária.

Caetano KS, et al. (2018) reiteram que as práticas educativas encabeçadas por profissionais da saúde, em especial pelo enfermeiro, empoderam os idosos na implementação de condutas de autocuidado e da corresponsabilização acerca do processo saúde-doença.

Outro estudo de Bezerra VP, et al. (2015) destacou que grande parte dos idosos pesquisados mantinha prática sexual desprotegida em seus relacionamentos estáveis, apegados à ideia de fidelidade do parceiro, 0 que evidencia falta de conhecimento ou ingenuidade. Na atual conjuntura, todos os sujeitos, independente da faixa etária, encontram-se em situação de vulnerabilidade às ISTs e ao HIV, tendo em vista que tais infecções não elegem raça, condição social, faixa etária ou gênero. E apesar da confiança na fidelidade do companheiro, algumas ISTs podem ser adquiridas antes do início da relação dita "fixa" ou "séria", e sua descoberta pode ocorrer apenas com o surgimento de sinais e sintomas tempos depois.

Quando apresentados a imagens ilustrativas das manifestações clínicas de ISTs como sífilis, gonorreia, infecção pelo papilomavírus humano (HPV), herpes genital e HIV/síndrome da imunodeficiência adquirida (AIDS) muitos idosos demonstram sentimentos de espanto, inquietude e repulsa. Por outro lado, esse pode ser um momento de identificação com o que foi exibido nas fotografias, servindo para os sujeitos relatarem experiencias vividas previamente (MOREIRA WC, et al., 2015).

De acordo com os resultados do estudo de Silva AT, et al. (2019) que verificou a prevalência da AIDS no Centro-Oeste brasileiro em um período de 10 anos, mostrou maior frequência de casos da doença na faixa etária de 60-69, adquirida por meio de relação heterossexual e em sujeitos do sexo masculino. Diante do cenário atual, Peixer TC, et al. (2015) evidenciam que o indivíduo idoso deve ter consciência da vulnerabilidade ao qual está exposto, podendo adquirir ISTs em relações sexuais desprotegidas. Esse trabalho de conscientização é frequentemente um desafio, exigindo atuação efetiva da Enfermagem na explanação dos riscos e no esclarecimento de dúvidas. O investimento em condutas voltadas à prevenção é primordial à sensibilização e à adoção de práticas sexuais seguras.

O estudo de Brito NMI, et al. (2016) investigou o conhecimento e analisou a percepção de idosos sobre o risco de transmissão das ISTs/HIV. Os autores ressaltaram que os idosos estão sujeitos, especificamente, à epidemia da AIDS existente no Brasil, apesar do trabalho incessante do Estado na divulgação de mensagens institucionais nos meios de comunicação. Existe certa resistência pelos sujeitos dessa faixa etária quanto ao uso do preservativo masculino e feminino, apesar do conhecimento das consequências da não utilização do recurso. É necessária abordagem do tema de forma aberta, livre de discriminação, proporcionando a manifestação da sexualidade, segurança e qualidade de vida.

Palestras e outras ações de educação em saúde emergem como estratégia útil na prevenção das ISTs pois, quando bem executadas, servem como espaços para debate e sessões de tira-dúvidas. $O$ desconhecimento de condutas simples, como a colocação do preservativo, é real e não deve ser encarado por atitudes condenatórias do profissional de saúde (MOREIRA WC et al., 2015). Em meio ao emprego dessas ações preventivas, os grupos de convivência de idosos podem ser de grande importância à prevenção das ISTs. Esses espaços são excelentes formas de socialização e troca de experiência e proporcionam ao profissional de saúde a chance de abordagem das diversas temáticas relacionadas ao envelhecimento e à disseminação de informações livres de preconceito na terceira idade (OLIVEIRA JMS e CÂNDIDO ASC, 2016). 
A sexualidade precisa ser discutida com os idosos e incentivada por meio de práticas saudáveis e sem estigmas, de maneira que favoreça uma vida independente e plena, com vistas a uma qualidade de vida satisfatória na terceira idade. É necessária a adoção de uma assistência integral sobre os diversos aspectos socioculturais aos quais o idoso está englobado. Compreende-se que existem fragilizações na prevenção das ISTs pelo idoso, contudo deve ser utilizado o conhecimento desses sujeitos sobre tais infecções para o fortalecimento da promoção da saúde a essa população (SILVA JDB, et al., 2017).

\section{CONSIDERAÇÕES FINAIS}

O estudo expõe déficits de conhecimentos da população idosa em relação à saúde sexual e à prevenção de infecções sexualmente transmissíveis. Pode-se inferir que os processos educativos ofertados a essa demanda da sociedade não estão sendo implementados corretamente. A Enfermagem, por ser uma profissão que lida com o paciente nos três níveis de atenção à saúde, deve adotar estratégias de ações educativas singulares apropriadas ao grau de conhecimento do idoso que está sendo atendido, de modo a construir uma discussão baseada nas informações prévias que o sujeito possui acerca das infecções. Acolhimento, criação de vínculo, atendimento holístico e diálogo emergem como estratégias importantes na edificação de uma relação profissional-paciente de confiança, possibilitando adesão às práticas orientadas pelo profissional de Enfermagem.

\section{REFERÊNCIAS}

1. ANDRADE J, et al. Vulnerabilidade de idosos a infecções sexualmente transmissíveis. Acta Paulista de Enfermagem, 2017; 30(1): 8-15.

2. ASSIS CL, FILHO JS. Sexualidade na terceira idade: estudo a partir de um grupo de idosos de uma associação do interior de Rondônia. Políticas e Saúde Coletiva, 2015; 1(2): 199-213.

3. BEZERRA VP, et al. Práticas preventivas de idosos e a vulnerabilidade ao HIV. Revista Gaúcha de Enfermagem, 2015; 36(4): 7076.

4. BEZERRA VP, et al. Vulnerabilidade de idosos ao contágio pelo HIV no contexto de práticas preventivas. Revista de Enfermagem da UFPE, 2014; 8(1): 22-29.

5. BRITO NMI, et al. Idosos, infecções sexualmente transmissíveis e aids: conhecimentos e percepção de risco. ABCS Health Sciences, 2016; 41(3):140-145.

6. CAETANO KS, et al. HIV/AIDS: conhecimento, atitude e prática da pessoa idosa. Itinerarius Reflectionis, 2018; 14(4): 1-21.

7. CASSÉTTE JB, et al. HIV/aids em idosos: estigmas, trabalho e formação em saúde. Revista Brasileira de Geriatria e Gerontologia, 2016; 19(5): 733-744.

8. CASTRO SFF, et al. Prevenção da AIDS em idosos: visão e prática do enfermeiro. Ciência \& Saúde, 2014; 7(3): 131-140.

9. CUNHA LM, et al. Vovó e vovô também amam: sexualidade na terceira idade. Revista Mineira de Enfermagem, 2015; 19(4): 894900.

10. FIGUEIROA MN, et al. A formação relacionada com a sexualidade humana na percepção de estudantes de enfermagem. Revista de Enfermagem Referência, 2017; 4(15): 21-30.

11. MALAQUIAS BSS, et al. Pesquisa com idosos sobre HIV/AIDS e sexualidade: relato de experiência. REFACS, $2017 ; 5(2): 255-262$.

12. MINICHIELLO V, et al. STI epidemiology in the global older population: emerging challenges. Perspect Public Health, 2012; 132(4): 178-181.

13. MOREIRA WC, et al. Sexualidade e prevenção de IST e HIV/aids entre idosos usuários da estratégia saúde da família. Revista Prevenção de Infecção e Saúde, 2015; 1(3): 76-82.

14. OLIVEIRA JMS, CÂNDIDO ASC. Conhecimento dos Idosos sobre as medidas de prevenção das DST's. Revista Multidisciplinar e de Psicologia, 2016; 10(31): 154-165.

15. PEIXER TC. Sexualidade na terceira idade: percepção de homens idosos de uma estratégia de saúde da família. Journal of Nursing and Health, 2015; 5(2): 131-140.

16. SILVA AT, et al. Prevalência da AIDS em idosos no centro-oeste brasileiro. Revista Eletrônica Acervo Saúde, $2019 ;$ (23): e434.

17. SILVA JDB, et al. Vulnerabilidade às infecções sexualmente transmissíveis/AIDS em idosos. Revista UNINGÁ, $2017 ; 53(1)$ : $19-24$.

18. SOUZA MP. A percepção dos idosos sobre a sexualidade: revisão sistemática da literatura. Saúde \& Transformação Social, 2015; 6(1): 124-131.

19. VIEIRA KFL, et al. A sexualidade na velhice: representações sociais de idosos frequentadores de um grupo de convivência. Psicologia: Ciência e Profissão, 2016; 36(1): 196-209. 